\title{
ANÁLISE DOS MOTIVOS PARA USO DO SERVIÇO DE URGÊNCIA E EMERGÊNCIA PELO PACIENTE NÃO GRAVE
}

\author{
ANALYSIS OF THE REASONS WHY NON-URGENT PATIENT SEEK \\ EMERGENCY DEPARTMENT.
}

Prof. Dra. Silvania Klug Pimentel1; Melissa Mello Mazepa2; Mariana Collodetto Soares²; Paulo
Victor Kioshima Kato2; Rebeca Ludmila de Lima2; Maryella Eduarda Correa Soares2;

\section{RESUMO}

OBJETIVO: Este estudo tem como objetivo reconhecer os motivos pelos quais pacientes que possuem problemas de saúde não graves procuram atendimento médico em serviço de Pronto Socorro (PS), não optando pelos serviços de Unidades Básicas de Saúde (UBS) ou Unidades de Pronto Atendimento (UPA) 24 horas. MÉTODOS: A pesquisa foi aprovada pelo Comitê de Ética em Pesquisa em Seres Humanos do Hospital do Trabalhador. Foi aplicado um questionário objetivo em uma amostra aleatória de 197 pacientes classificados como não urgentes ou pouco urgentes segundo o Manchester Triage System (MTS). RESULTADOS: Dos pacientes entrevistados 65\% eram homens. Apenas $47 \%$ entendiam a real função do PS. Sobre o MTS, $52 \%$ conheciam o sistema e sabiam que seriam triados, mas mesmo assim a maioria destes veio buscando melhor resolutividade para seus problemas. Mesmo após esclarecimento sobre a função do PS em atender os casos graves, $73 \%$ afirmaram que teriam vindo da mesma forma ao PS e 83\% consideram a aplicação do MTS um sistema justo de triagem. CONCLUSÃO: Concluímos que não é somente a falta de informação que faz pacientes não graves se direcionarem ao PS, mas sim a falta de confiança na capacidade resolutiva da UBS e UPA 24 horas.

Descritores: Triagem, Protocolo de Manchester, Pronto Socorro, Emergência, Política de Saúde.

\section{ABSTRACT}

OBJECTIVE: The objective of this study was to identify the reasons why non-urgent patients use emergency department instead the Basic Health Units. METHODS: An objective questionnaire was applied in a random sample of 197 patients classified as non-urgent by Manchester Triage System (MTS). RESULTS: Of the patients surveyed $65 \%$ were men. Only $47 \%$ understood the real function of the emergency department. About MTS, 52\% knew the triage system and knew they would be screened, yet most of these came looking for a better resolution for their medical problems. Even after clarification about the role of emergency department, that is to resolve severe cases, $73 \%$ said they would have gone to the emergency department either way and $83 \%$ consider the application of MTS a fair system of screening. CONCLUSION: We conclude that it is not only the lack of information that makes non-urgent patients seek the emergency department, but the lack of confidence in the response capacity of Basic Health Units.

Keywords: Triage System, Manchester Triage System, Emergency Department Emergency, Health Policy.

1 - Professora do Departamento de Cirurgia da Universidade Federal do Paraná, Cirurgiã do trauma do Hospital do Trabalhador.

2 - Acadêmico de Medicina, Universidade Federal do Paraná.

Contato do Autor / Mail to:

Melissa Mello Mazepa - melissa.mazepa@gmail.com

Rua Humberto de Campos, 446, Pilarzinho, Curitiba - PR, CEP: 82120-410 


\section{INTRODUÇÃO}

Em serviços de urgência e emergência o atendimento rápido e eficaz é fundamental para evitar a morte de pacientes graves ${ }^{1}$. Para facilitar o manejo dos pacientes que chegam aos centros de referência foi criado - Manchester Triage System (MTS) ${ }^{2}$, sistema de classificação de risco que consiste em categorizar os pacientes em cinco níveis de prioridade de atendimento de acordo com a queixa principal e avaliação clínica feita na triagem e pré-determinar um tempo máximo de atendimento adequado à gravidade de cada grupo ${ }^{1}$ (Figura 1).

A unidade de emergência do Hospital do Trabalhador é referência para o atendimento de vítimas graves de trauma na cidade de Curitiba, que normalmente chegam ao hospital via sistema de ambulância. Entretanto, também é um hospital que atende pacientes por procura espontânea. Em 2012 foram atendidos 63855 pacientes no pronto socorro (PS) deste hospital, somando pacientes graves e não graves, com uma média de 177 pacientes por

\begin{tabular}{|c|l|c|c|}
\hline & \multicolumn{1}{|c|}{ Prioridade } & COR & TEMPO \\
\hline 1 & Emergente & Vermelho & 0 minutos \\
\hline 2 & Muito Urgente & Laranja & 10 minutos \\
\hline 3 & Urgente & Amarelo & 60 minutos \\
\hline 4 & Pouco Urgente & Verde & 120 minutos \\
\hline 5 & Não Urgente & Azul & 240 minutos \\
\hline
\end{tabular}

dia $^{3}$.

Figura 1: Protocolo de Manchester. Níveis de gravidade por cores e tempo esperado para atendimento.

De acordo com a literatura internacional estimase que pacientes não urgentes que se dirigem ao pronto socorro estão entre 33\% a 50\% dos atendimentos ${ }^{4}$. Alguns autores acreditam que a grande procura por atendimento por estes pacientes é a principal causa de superlotação, o que geraria resultados adversos para pacientes que estão gravemente enfermos 5,6 . Além disso, o encaminhamento proveniente de Unidades Básicas de Saúde (UBS) ou Unidades de Pronto Atendimento 24 horas (UPA) também figura entre as causas de superlotação do pronto-socorro.

No Brasil foi desenvolvida em 2003, pelo Ministério da Saúde, a Política Nacional de Urgência e Emergência, visando evitar que pacientes de baixa e média complexidade sejam encaminhados aos prontos-socorros dos hospitais. As UBS têm como prioridade o atendimento das urgências de menor complexidade. As UPAs funcionam como unidades intermediárias para atendimento das urgências de média complexidade (como febre alta, fraturas, cortes e infartos). O setor de urgência dos hospitais é destinado ao atendimento de maior complexidade por meio do atendimento em pronto socorro, unidade destinada à prestação de assistência a pacientes com ou sem risco de vida, cujos agravos necessitam de atendimento imediato 7,8 .
Tem-se claro na literatura que os PS são muito utilizados como porta de entrada ao sistema de saúde pelos pacientes não graves.

Assim o objetivo deste estudo é reconhecer os motivos pelos quais pacientes que possuem problemas de saúde não graves procuram atendimento médico em serviço de PS, não optando pelos serviços de Unidades Básicas de Saúde ou Unidades de Pronto Atendimento 24 horas.

\section{MÉTODOS}

Realizou-se estudo observacional, analítico e transversal. Participaram da pesquisa 197 pacientes que procuraram espontaneamente o pronto atendimento do Hospital do Trabalhador (HT) compondo uma amostra por conveniência e aleatória. O HT localiza-se no município de Curitiba e trata-se de um Hospital de grande porte, referência em assistência de urgência e emergência traumatológica.

O recrutamento dos indivíduos se deu através de convite oral à participação da pesquisa. Foram incluídos no estudo pacientes classificados de acordo com o Protocolo de Manchester em não urgentes ou pouco urgentes e que aceitaram participar como também assinar o termo de consentimento livre e esclarecido. Não houve critérios de exclusão.

Os participantes responderam um questionário composto por sete perguntas fechadas, além de idade e gênero, elaborado e aplicado pela equipe de pesquisadores. 0 conjunto de questões foi lançado por estes em uma plataforma virtual do Google docs ${ }^{\circledR}$ e os mesmos preencheram-na com as respostas fornecidas pelos pacientes. Os questionários foram aplicados na sala de espera do pronto atendimento do Hospital do Trabalhador durante o intervalo entre a realização da triagem feita de acordo com o Protocolo de Manchester e o atendimento médico.

A sistematização dos dados foi feita por meio de análise da frequência de resposta de cada pergunta do questionário supracitado com o auxílio do programa Microsoft Excel.

O projeto foi submetido ao Comitê de Ética em Pesquisa (CEP) do Hospital do Trabalhador, sendo então aprovado através do parecer n 43169915.4.0000.5225.

\section{RESULTADOS}

O estudo contou com a participação de 197 pacientes, sendo a maioria $65 \% \quad(n=128)$ do sexo masculino. A média de idade foi de 32,3 anos ( $D P \pm 17,5$ ) e a mediana de 28 anos.

Em relação à especialidade procurada pelos pacientes, o serviço de ortopedia atendeu $77,2 \%(n=152)$ 
dos pacientes, o serviço de cirurgia geral atendeu 14,7\% $(n=29)$ dos pacientes, $7,1 \% \quad(n=14)$ necessitavam de atendimento em ambos os serviços e 1\% $(n=2)$ dos pacientes buscaram o serviço de clínica médica.

Quando questionados sobre a função do PS, $47,2 \%(n=93)$ acreditam que corresponde a atender casos mais graves, de maior risco à vida, 42,1\% $(n=83)$ dos pacientes responderam que a função do PS é atender todos os tipos de consultas e $7,1 \%(n=14)$ dos pacientes tem outra opinião a respeito da função do PS.

No que concerne aos motivos que levaram o paciente a se dirigir para o pronto socorro e não à UBS ou a UPA, a maioria, 30,5\% $(n=60)$, relatou busca por maior resolutividade (Tabela 1). Setenta e sete pacientes $(39,1 \%)$ listaram outros motivos para buscarem atendimento no PS, sendo que $21,8 \%$ relataram que foram encaminhados ao PS pelos serviços das UBS ou pela UPA (Tabela 2 ).

Tabela 1: Resposta à pergunta "Por que veio ao Pronto

\begin{tabular}{lcc}
\hline Respostas & N & Frequência \\
\hline Mais próximo e fácil & 16 & $8,1 \%$ \\
Maior Resolutividade & 60 & $30,5 \%$ \\
Mais rápido & 20 & $10,2 \%$ \\
Desconhece a função do pronto socorro & 13 & $6,6 \%$ \\
Outros & 77 & $39,1 \%$ \\
\hline N: amostra & &
\end{tabular}

Socorro e não a UBS?"

Sobre o serviço de triagem realizado no hospital e a existência da classificação de risco dos pacientes, 51,8\% $(n=102)$ dos entrevistados afirmaram conhecer o sistema, enquanto $48,2 \% \quad(n=95)$ não tinham conhecimento. Daqueles que tinham conhecimento sobre a existência da classificação de risco e vieram ao PS da mesma maneira, quase $45 \%$ desses acharam que a UBS não resolveria seus problemas. Outros motivos que fizeram com que pacientes não graves fossem ao PS mesmo sabendo da classificação de risco estão ilustrados na tabela 3.

Tabela 2: Resposta à pergunta “Por que veio ao Pronto

\begin{tabular}{lcc}
\hline Respostas & N & Frequência \\
\hline Encaminhados pela UBS ou UPA & 43 & $21,8 \%$ \\
$\begin{array}{l}\text { Encaminhado pela empresa ou acidente } \\
\text { de trabalho }\end{array}$ & 18 & $9,13 \%$ \\
Trazido por SIATE/SAMU & 2 & $1 \%$ \\
Outros & 14 & $7,1 \%$ \\
\hline N: amostra & & \\
\hline
\end{tabular}

Socorro e não a UBS?" Representação de "Outros".

Ainda sobre a classificação de risco $84,8 \%$ $(n=167)$ dos pacientes acham justo esse tipo de classificação, ao passo que $13,2 \% \quad(n=26)$ não a consideram justa.

Após reiterar se tratarem de casos não graves e esclarecimento sobre a função do pronto socorro, 73,1\% $(n=144)$ relataram que teriam se dirigido ao pronto socorro da mesma maneira. 0 restante declarou que teria optado pela UBS.

Tabela 3: Resposta à pergunta: “Mesmo sabendo da existência da classificação de risco, por que vieram ao

\begin{tabular}{lll}
\hline Respostas & N & Frequência \\
\hline Achou que a UBS não resolveria seu problema & 44 & $43,13 \%$ \\
Achou que seria classificado como mais grave e & 7 & $6,8 \%$ \\
teria prioridade & 8 & $7,8 \%$ \\
Não se importa em esperar & 39 & $38,23 \%$ \\
Outros & & \\
\hline N: amostra & &
\end{tabular}

Pronto Socorro?"

\section{DISCUSSÃO}

Em relação ao perfil do paciente não urgente que se dirigiu espontaneamente ao PS durante o estudo, 65\% era do sexo masculino e a média de idade foi de 32,3 anos. Northington et al. encontrou resultado semelhante, com a maioria de homens $(53,3 \%)$ e uma média de idade de 36,1 anos 4 . Esse achado é corroborado por estatísticas nacionais que referem um maior acometimento do homem em causas externas, com consequente maior procura ao pronto atendimento?.

Verificamos que o principal motivo pelo qual os pacientes não urgentes se dirigem ao pronto socorro em detrimento da UBS ou UPA é maior resolutividade do PS. De maneira semelhante, em estudo conduzido por Northington et al. $76,1 \%$ dos pacientes citaram que o pronto socorro seria o melhor local para busca de atendimento, por este ser mais resolutivo ${ }^{4}$. Outra característica apontada em nosso estudo foi o fato de o PS ser mais rápido, atributo apontado por $68,6 \%$ dos pacientes no estudo realizado por Northington et al. 4 .

Encontramos como a segunda causa mais prevalente de busca ao PS pelo paciente não grave o encaminhamento realizado pela atenção básica à saúde. Em estudo canadense realizado por Burnett et al. os pacientes encaminhados atingiram $40 \%$ dos pacientes não urgentes $^{10}$. Isso demonstra que além de uma porção substancial dos pacientes se dirigirem ao pronto 
atendimento mesmo com existência de alternativas na comunidade, uma parcela dos pacientes não urgentes seguiu recomendação médica para tal busca.

As UBS devem ser capazes de atender urgências de baixa complexidade, de maneira resolutiva, evitando encaminhamentos desnecessários aos centros de complexidade terciária, particularmente, hospitais de maior porte, possibilitando que os leitos sejam ocupados por usuários que realmente deles necessitem ${ }^{11,12}$. Uma vez que o presente estudo encontrou que mais de um quinto dos usuários que buscaram atendimento no PS na realidade foram encaminhados pela atenção básica e secundária, pode-se inferir que ocorrem encaminhamentos desnecessários de pacientes não urgentes de maneira significativa.

O sistema de triagem de Manchester classifica os pacientes em cinco grupos de urgência, que podem ser separados em dois grupos maiores, os urgentes (cores vermelha, laranja e amarela) e não urgentes (verde e azul). Nosso estudo mostrou que grande maioria dos pacientes não urgentes acha justo esse sistema de triagem e que ela deve ser realizada priorizando o atendimento daqueles mais graves.

A informação obtida nesta pesquisa de que dentre os entrevistados que conheciam a Classificação de Risco, 45\% acreditava que a atenção básica e secundária não resolveria seu problema nos aponta para a indispensabilidade do conhecimento sobre as atribuições das UBS e UPA, estabelecendo-se como primordial para que o usuário busque atendimento em local apropriado e, para que tais Unidades não sejam desacreditadas. Adicionalmente, a desvalorização dessas unidades pela população surge como uma das razões da busca pelo paciente não grave ao Pronto Socorro13.

Para Garlet et al. os recursos oferecidos por Emergências Hospitalares, tais como consultas, remédios, procedimentos de enfermagem e internações tornam-nas resolutivas na visão do usuário, que passa então a desconsiderar a superlotação e a impessoalidade do atendimento prestado ${ }^{14}$. Tais fatos corroboram e justificam os dados deste estudo que revelam que quando questionados sobre o motivo de terem procurado um PS e não uma UBS ou UPA 24horas, a maioria respondeu que pretendia obter maior resolutividade. E após serem esclarecidos sobre a real função do PS 73,1\% afirmaram que ainda assim procurariam esse mesmo local para solucionar sua queixa.

Esses resultados estão em concordância com estudos brasileiros, nos quais também foi demonstrado alta proporção de pacientes com queixas não urgentes e teoricamente passíveis de serem resolvidos em unidades intermediarias procurando serviços de urgência15,16. A grande demanda nos serviços de PS também está relacionada à confiança dos usuários na assistência prestada pelo hospital somada a problemas da rede básica - tanto de resolutividade quando outros fatores como dificuldade para marcar consulta na UBS, ausência da necessidade de agendamento de consulta ou maior disponibilidade de recursos no serviço de pronto atendimento ${ }^{17,18}$. Os resultados do presente estudo, assim como a literatura nacional, mostram uma tendência hospitalocêntrica que continua sendo uma barreira a ser vencida nos serviços de saúde17,18.

0 desencontro entre o conceito de urgência e emergência adotado pelo paciente e aquele incorporado pelo modelo tradicional biomédico, utilizado nas unidades de atendimento a urgências e emergências também é fator determinante para a procura por atendimento em local inadequado ${ }^{14,19}$. Em nosso estudo $6,8 \%$ dos pacientes que tinham conhecimento do MTS acharam que seriam classificados como mais graves. Dessa maneira, pode-se afirmar que a visão distorcida sobre seu próprio quadro fez com que alguns pacientes buscassem o pronto socorro erroneamente. Isso demonstra a necessidade de informar e educar a sociedade a fim de que a auto percepção da doença leve à procura de uma unidade apropriada para satisfazer suas necessidades.

Assim, demonstra-se que somente a implantação da Classificação de Risco de Manchester não assegura uma melhoria na qualidade da assistência primária, secundária e terciária enquanto unidades formadoras de um sistema único de saúde, tornando-se essencial a construção de acordos e tratos internos e externos para a viabilização do processo, com a construção de fluxos claros por grau de risco e a garantia de sua efetivação na rede de atenção20.

Observa-se uma falha no fluxo de atendimento dos serviços de saúde, sendo que serviços de urgências e emergências são sobrecarregados pelo volume muito grande de casos de complexidade menor que poderiam ser atendidos nas unidades básicas ${ }^{4}$. É necessária uma intervenção dos gestores visando maior informação à população acerca de onde procurar o atendimento, além de tornar a atenção primária mais efetiva e resolutiva. Estas informações poderão ajudar a mapear as necessidades de atendimento de saúde da população de Curitiba e assim contribuir para otimizar o atendimento no serviço e nas unidades básicas de saúde.

\section{CONCLUSÃO}

O presente estudo mostra que não é somente a falta de informação sobre a real função de um pronto socorro que faz os pacientes não graves se direcionarem ao pronto atendimento, mas também a falta de confiança na capacidade resolutiva da UBS e UPA 24 horas. Além disso, a alta taxa de encaminhamentos pelas unidades intermediárias contribui de forma significativa para a superlotação.

Os dados podem servir para planejar um cuidado de qualidade direcionado para as necessidades de saúde da população em serviços de urgência e emergência. É necessária maior atenção dos gestores de saúde para orientação da população, esclarecendo sobre os diferentes serviços de saúde e suas funções e no planejamento de ações que visem à resolutividade da Atenção Primária, que 
terá como consequência a diminuição da superlotação do pronto atendimento e do tempo de espera, além de melhorar a qualidade de atendimento.

\section{CONFLITOS DE INTERESSE}

Não houve conflitos de interesse.

\section{REFERÊNCIAS}

1. Grupo Brasileiro de Classificação de Risco. Diretrizes para implementação do Sistema Manchester de Classificação de Risco nos pontos de atenção às urgências e emergências Segunda Edição 2015 [Internet]. Grupo Brasileiro de Classificacao de risco.; 2015. Available from: http://www.gbcr.org.br/downloads.

2. Manchester Triage Group. Emergency Triage. John Wiley \& Sons; 2008. 195 p.

3. Hospital do Trabalhador [homepage na internet]. Tipos de Atendimento no Pronto Socorro [acesso em 16 maio 2015]. Disponível em: http:www.hospitaldotrabalhador.saude.pr.gov.br

4. Northington WE, Brice JH, Zou B. Use of an emergency department by nonurgent patients. Am J Emerg Med. 2005 Mar;23(2):131-7.

5. Lowe RA, Bindman AB, Ulrich SK, Norman G, Scaletta $T A$, Keane $D$, et al. Refusing care to emergency department of patients: evaluation of published triage guidelines. Ann Emerg Med. 1994 Feb;23(2):28693.

6. Li G, Grabowski JG, McCarthy ML, Kelen GD. Neighborhood characteristics and emergency department utilization. Acad Emerg Med. 2003 Aug;10(8):853-9.

7. Portal Brasil. Unidades de Saúde ajudam a desafogar prontos-socorros [Internet]. Portal Brasil; 2011 [atualizado em 2014 Jul 28; citado em 2015 Maio 11]. Disponivel em: http://www.brasil.gov.br/saude/2011/08/unidadesajudam-a-desafogar-os-prontos-socorros.

8. Portal da Saúde. Informações de Saúde - tipos de estabelecimento [Internet]. Brasília: Ministério da Saúde, Departamento de Informática do SUS; [atualizado em 2006 Set 28; citado em 2015 Maio 11]. Disponivel em: http://tabnet.datasus.gov.br/cgi/cnes/tipo_estabele cimento.html

9. Gawryszewski VP, Koizumi MS, Mello Jorge MHP. As causas externas no Brasil no ano 2000: comparando a mortalidade e a morbidade. Cad Saúde Pública. 2004;20:995-1003.

10. Burnett $M$, Grover S. Use of the emergency department for nonurgent care during regular business hours. CMAJ. 1996;154:1345- 51.

11. Soares SS, Lima LDD, Castro ALBDO. O papel da atenção básica no atendimento às urgências: um olhar sobre as políticas. J Manag Prim Health Care. 2014;5(2):170-7.

12. Erdman AL, Andrade SR, Mello ALSF, Drago LC. A atenção secundária em saúde: melhores práticas na rede de serviços. Rev Latino-Am Enfermagem. 2013; 21:131- 139.

13. Nascimento APS, Santos LF, Carnut L. Atenção primária à saúde via estratégia saúde da família no Sistema Único de Saúde: uma introdução sobre os problemas inerentes à operacionalização de suas ações. J Manag Prim Health Care. 2011;2(1):18-24.

14. Garlet ER. Lima MADS, Santos JLG, Marques GQ. Finalidade do trabalho em urgências e emergências. Rev. Latino-Am. Enfermagem. 2009;17(4):535-540.

15. Puccini PT, Cornetta VK. Ocorrências em prontosocorro: eventos sentinela para o monitoramento da atenção básica de saúde. Cad Saude Publica. 2008;24(9):2032-42.

16. Pires MRGM. Göttems LBD, Cupertino TV, Leite LS, Vale RL, Castro MA et al. A utilização dos serviços de atenção básica e de urgência no SUS de Belo Horizonte: problema de saúde, procedimentos e escolha dos serviços. Saúde Soc. 2013;22(1):211-22

17. Dubeux LS, Freese E, Felisberto E. Acesso a hospitais regionais de urgência e emergência: abordagem aos usuários para avaliação do itinerário e dos obstáculos aos serviços de saúde. Physis. 2013;23(2):345-69

18. Caccia-Bava MCG, Pereira MJB, Rocha JSY, Martinez EZ. Pronto-atendimento ou atenção básica: escolhas dos pacientes no SUS. Medicina (Ribeirão Preto). 2011;44(4):347-54.

19. Gifford MJ, Franaszek JB, Gibson G: Emergency physicians' and patients' assessments: urgency of need for medical care. Ann Emerg Med. 1980;9:502 7.

20. Abreu KP. Ministério da Saúde. Secretaria de Atenção à Saúde. Política Nacional de Humanização da Atenção e Gestão do SUS. Acolhimento e Classificação de Risco nos Serviços de Urgência. Brasília: Ministério da Saúde, 2009b. (Série B. Textos Básicos de Saúde). 